\title{
Nanoparticle Photochemistry via Nano-Impacts
}

Thomas R. Bartlett, Stanislav V. Sokolov and Richard G. Compton*

Department of Chemistry, Physical and Theoretical Chemistry Laboratory, Oxford University, South Parks Road, Oxford, OX1 3QZ, United Kingdom

KEYWORDS: Nanoparticle, nano-impacts, photochemistry, voltammetry, silver bromide.

ABSTRACT: We report the use of nano-impacts as a novel method for the study of photochemical reactions of individual nanoparticles (NPs). The conversion of gelatine stabilised silver bromide (AgBr) NPs to silver (Ag) NPs through photochemical reduction by ascorbic acid is studied mechanistically. Two mechanisms are proposed and investigated by monitoring the amount of electrochemically accessible $\mathrm{AgBr}$ against the time scale of conversion, measured through the use of the nano-impacts technique. 


\section{INTRODUCTION}

The development of new solution-based synthesis methods for nanoparticles (NPs), especially silver (Ag), is important for the continued advancement in areas such as biology [1] and analysis [2]. Established methods for solution-based NP synthesis have revolved around the "bottom-up" approach [3], encompassing techniques such as chemical reduction [4-6] and electrodeposition [7]. The mechanism of such solution-based synthesises have been thoroughly studied [8, 9] and originate with the formation of "seed" particles. With continued reaction, these "seeds" begin to nucleate, and grow by Ostwald ripening to form nanostructures, which are stabilised with a capping agent.

However, an alternative method has been developed that does not directly fit into this category - direct conversion. Zhu et al showed it was possible to chemically alter pre-existing titanate nanofibers, post-synthesis, through wet-chemistry phase transitions to titanium dioxide [10]. Other studies have also demonstrated the ability to take stable spherical NPs and alter their structure through chemical reactions [11]. More recently however, the chemical conversion of spherical NPs was achieved whilst maintaining the spherical structure of the pre-cursor NP. This novel synthesis method has been realised through the photochemical reduction of gelatine stabilised silver bromide (AgBr) NPs to silver (Ag) NPs [12, 13]. This new and attractive direction for the synthesis of NPs opens new avenues for NP design. However, little is understood about the mechanism for this conversion, especially for the photochemical conversion of spherical particles.

In this paper we consider two different mechanisms for the photochemical conversion of NPs, using the photochemical reduction of AgBr NPs by ascorbic acid as a model. First, is the "coreshell” mechanism (see Scheme 1(i)), whereby growth initiates evenly across the surface of the 
particle from the latent sites, forming a shell of Ag. This shell might limit the migration of $\mathrm{Br}^{-}$ and release of the halide, halting the conversion before the AgBr core can be converted [14]. Secondly is the "irregular growth" mechanism (see Scheme 1(ii)). Here the conversion propagates from random sites on the NP. This might allow the core of the particle to become converted before the process is deactivated through the complete reaction of the surface.

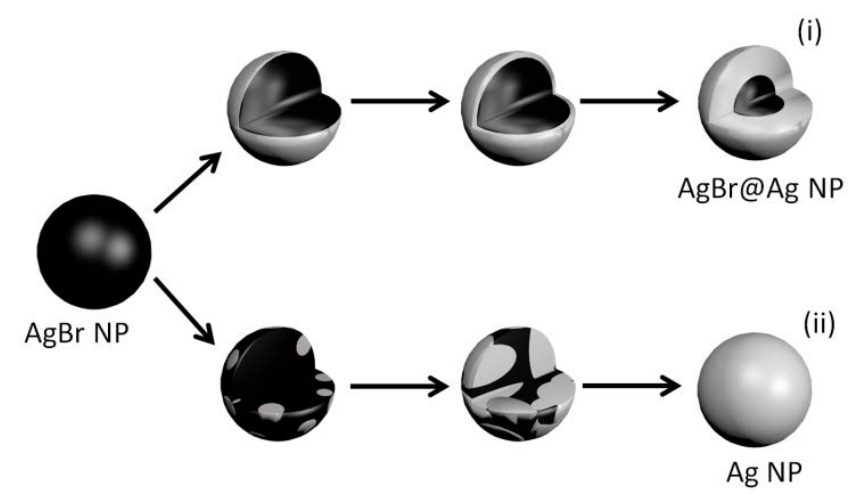

Scheme 1. Proposed pathways for the photochemical conversion of a Ag NP by ascorbic acid. (i) "Core-shell” method whereby conversion progresses evenly across the whole surface towards the centre of the NP. (ii) "Irregular" conversion resulting in a complete Ag NP

In this paper the possible mechanism will be electrochemically probed using the nano-impacts technique [15]. This technique has not previously been used to study the synthesis of particles however the method inherently lends itself to the mechanistic study of such conversions. The development of this technique has resulted in two main classifications of analytical detection: mediated and direct nano-impacts. Mediated impacts involve the measurement of faradaic currents as a result of the catalysis of solution based redox active species at the NP surface. This occurs only when the NP resides within electron-tunnelling distance of the electrode, and has been demonstrated by the electrochemical oxidation of hydrazine on platinum NPs [16], the photoelectrochemical oxidation of methanol by titanium oxide NPs [17] and more recently by 
the platinum NP mediated reduction of ferrocyanide on $\mathrm{TiO}_{2}$ passivated n-type silicon electrodes [18].

The more important technique relating to the work reported herein is the direct nano-impact method. Here the redox activity of the NP material itself is utilised and was first demonstrated by the direct oxidation of Ag NPs [15]. As the NP collides with an electrode held at a suitably oxidising or reductive overpotential, electron transfer occurs. This results in a faradaic "spike" in current. This spike is a combination of faradaic and capacitive contributions however the latter component is negligible. If complete conversion of the particle occurs upon impact, the charge under each spike can then be directly related to the volume of the particle. This nano-impact method has been shown to give quantitative sizing for NPs of diameters from 4 to $100 \mathrm{~nm}$ [19, 20]. The amount of charge per NP collision therefore provides information on the amount of electrochemically accessible redox material available in individual NPs. If a shell of Ag is formed as in mechanism (i), it can be theorised that this would provide a barrier to electrochemical reduction of the AgBr. If an irregular conversion were to occur as in mechanism (ii), AgBr would be accessible to the electrode for the duration of the conversion. By studying this electrochemical availability of $\mathrm{AgBr}$ during the photochemical reaction, it is therefore possible to resolve the mechanism by studying the change over the timescale of photochemical conversion. This work extends the nano-impacts technique to the field of nanophotoelectrochemistry from a mechanistic perspective.

\section{EXPERIMENTAL}

Chemicals. Silver nitrate $\left(\mathrm{AgNO}_{3}, 99 \%\right)$, sodium bicarbonate $\left(\mathrm{NaHCO}_{3}, 99.7-100.3 \%\right)$ and ascorbic acid (99+ \%) were purchased from Sigma-Aldrich (Dorset, UK). Sodium bromide 
(NaBr, 99 \%) was supplied by M\&B laboratories (Sydney, Australia) and gelatine powder was purchased from VWR (Soulbury, UK). Sodium carbonate $\left(\mathrm{Na}_{2} \mathrm{CO}_{3}, 99.9 \%\right)$ was obtained from BDH Laboratory Supplies (Poole, UK). All the chemicals were used as received without further purification. Preparation of solutions was carried out using Millipore water of resistivity 18.2 $\mathrm{M} \Omega \mathrm{cm}$ at $298 \mathrm{~K}$ throughout. Sodium carbonate buffer was used to achieve a buffered solution with $\mathrm{pH}$ 10, and was measured using a $\mathrm{pH} 213$ Microprocessor $\mathrm{pH}$ Meter from Hanna Instruments (Leighton Buzzard, UK). Calibration of the $\mathrm{pH}$ meter was carried out using Hamilton Duracal Buffer standards purchased from Sigma-Aldrich.

Equipment. For electrochemical experiments, an Autolab PGSTAT 302N was used from Metrohm-Autolab (BV, Utrecht, Netherlands) fitted with an ECD module for reducing background noise for nano-impact experiments. A three-electrode system with a platinum wire counter and a saturated MSE reference electrode (equivalent to $+0.64 \mathrm{~V}$ vs normal hydrogen electrode, NHE) was used in all electrochemical experiments. These were purchased from Goodfellow Ltd. (Cambridge, UK) and BASi (West Lafayette, USA) respectively. The working electrode for stripping voltammetry was a $3.0 \mathrm{~mm}$ glassy carbon (GC) macrodisk supplied by $\mathrm{CH}$ Instruments (Austin, USA). For impact experiments a $7 \mu \mathrm{m}$ carbon fibre microelectrode from BASi was used. Prior to experimentation, electrodes were polished with $1,0.3$ and $0.05 \mu \mathrm{m}$ alumina powder supplied by Buehler (Coventry, UK). All experiments were conducted after degassing solutions with $\mathrm{N}_{2}$ and using a Faraday cage thermostated to $25^{\circ} \mathrm{C}$.

UV-Vis spectra were performed on a U-2001 spectrometer purchased from Hitachi (Mannheim, Germany) using $10 \mathrm{~mm}$ UV glass cells from Hellma Analytics (Essex, UK). The reference solution was that of the tested solution, without NPs. 
For photochemical conversion, a custom made glass cell with $22 \mathrm{~mm}$ diameter quartz glass bottom was constructed. Illumination was provided by a F-150 halogen lamp, WPI Ltd. (Hitchin, UK) fitted with a $2 \mathrm{ft}, 6.4 \mathrm{~mm}$ diameter glass fibre light guide purchased from Fiberoptics Technology, Inc. (Pomfret, USA) at $100 \%$ illumination. Distance between the cell window and the light guide was $20 \mathrm{~mm}$. White light illumination was used for all experiments (wavelengths 400-1200 nm).

Silver Bromide Nanoparticle Synthesis and Characterisation. Gelatine stabilised AgBr NPs were synthesised as previously reported in the literature [20]. Gelatine powder (0.75 g) was added to $10 \mathrm{~mL} \mathrm{H}_{2} \mathrm{O}$ and heated to $35^{\circ} \mathrm{C}$ in a $25 \mathrm{~mL}$ three-necked flask under constant stirring by magnetic stirrer at $350 \mathrm{rpm}$. Once at temperature, $5 \mathrm{~mL}$ of $2.0 \mathrm{M} \mathrm{AgNO}_{3}$ and $5 \mathrm{~mL}$ of $2.0 \mathrm{M}$ $\mathrm{NaBr}$ were simultaneously injected into the flask. The solution was allowed to stir for 5 mins whilst maintaining the reaction temperature at $35{ }^{\circ} \mathrm{C}$. After this the NP suspension was transferred to Faulken tubes and stored in the dark without additional purification steps. Synthesis was carried out under dark conditions to prevent deterioration of the particles.

The NP suspensions were sonicated for 5 seconds before being drop-cast on to a TEM-grid modified SEM sample holder. SEM images were then taken using a high-resolution SEM (LEO Gemini 1530, Zeiss, Jen, Germany). Figure 1 shows an image of the particles which were analysed using Image J public domain software. The NPs were found to be spherical with a radius of $(46 \pm 1) \mathrm{nm}$ with a standard deviation of $14 \mathrm{~nm}$. Here and throughout this paper the error value quoted is the error of the mean. 


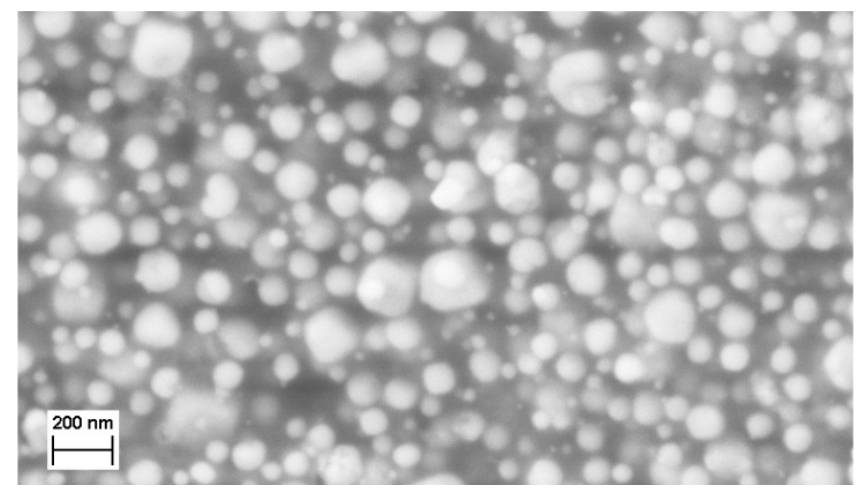

Figure 1. SEM image of gelatine stabilised AgBr nanoparticles.

\section{RESULTS AND DISCUSSION}

Prior to nano-impact investigation the voltammetric behaviour of an ensemble of NPs drop cast on to an electrode surface was studied in the chosen electrolyte - pH10 sodium carbonate buffer. This medium was used as alkaline conditions have been shown to slow down the photochemical reaction of $\mathrm{AgBr}$ with ascorbic acid [12], allowing the conversion to be studied over a suitable timescale for voltammetric study. Figure 2 shows the stripping voltammetry of 20 nmoles $\mathrm{AgBr}$ NPs drop cast on a GC electrode, in $0.10 \mathrm{M} \mathrm{pH} 10$ buffer, initially scanned reductively.

Reductive features, indicative of AgBr reduction can be observed after -0.75 V vs MSE:

$$
\operatorname{AgBr}(\mathrm{s})+\mathrm{e}^{-} \rightarrow \operatorname{Ag}(\mathrm{s})+\mathrm{Br}^{-}(\mathrm{aq})
$$

A double oxidation peak at $-0.10 \mathrm{~V}$ and $-0.01 \mathrm{~V}$ vs MSE can also be seen, and can be attributed to the oxidation of $\mathrm{Ag}$ to $\mathrm{AgBr}$ and $\mathrm{Ag}^{+}$respectively.

$$
\begin{aligned}
& \operatorname{Ag}(\mathrm{s}) \rightarrow \mathrm{Ag}^{+}(\mathrm{aq})+\mathrm{e}^{-} \\
& \mathrm{Ag}(\mathrm{s})+\mathrm{Br}^{-}(\mathrm{aq}) \rightarrow \operatorname{AgBr}(\mathrm{s})+\mathrm{e}^{-}
\end{aligned}
$$


This voltammetry is in agreement with that reported previously in $0.10 \mathrm{M} \mathrm{NaNO}_{3}$ electrolyte [20] and implies similar or greater potentials can likely be used for quantitative nano-impact experiments.

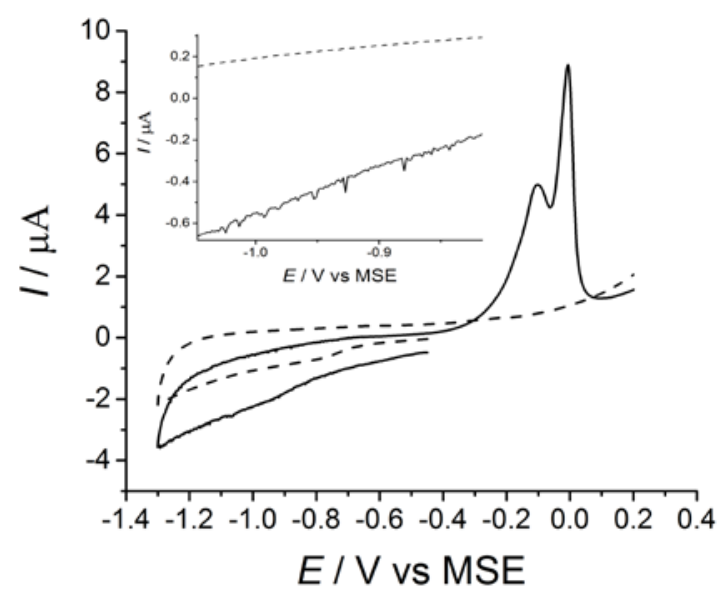

Figure 2. Cyclic voltammogram of a GC macro-electrode in $0.10 \mathrm{M} \mathrm{pH} 10$ buffer at $100 \mathrm{mVs}^{-1}$. Bare GC (dashed line). GC modified by drop-casting 20 nmoles of AgBr NPs (solid line). Insert shows a zoomed in image of the voltammogram and the reductive features observed only in the presence of the drop-cast NPs.

Next, nano-impact analysis of the AgBr NPs was conducted using a suspension of AgBr NPs in $0.10 \mathrm{M} \mathrm{pH} 10$ buffer $([\mathrm{AgBr}]=0.10 \mathrm{mM})$. A carbon fibre microelectrode was held at a reductive overpotential of $-1.00 \mathrm{~V}$ vs MSE and reductive faradaic nano-impact spikes were observed, as seen in Figure 3. 


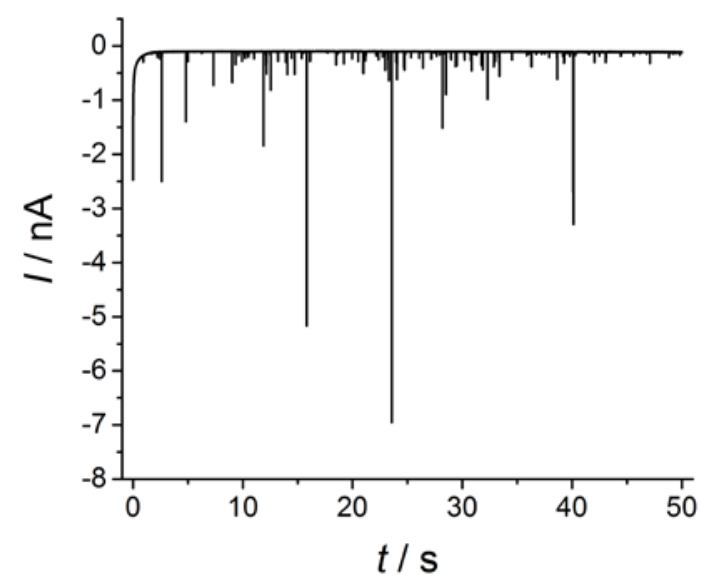

Figure 3. Typical current-time transient showing AgBr reductive impacts at $-1.00 \mathrm{~V}$ vs MSE. This was conducted in $0.10 \mathrm{mM}$ AgBr NP suspension and $0.10 \mathrm{M}$ pH10 buffer.

These spikes were analysed noting the spherical shape of the NPs to produce a size distribution using the equation [21]:

$$
R_{N P}=\sqrt[3]{\frac{3 M_{w} Q}{4 n F \pi \rho}}
$$

where $R_{N P}$ is the spherical NP radius, $M_{w}$ is the molecular weight of the NP material, $Q$ is the charge of the measured impact spike, $\mathrm{n}$ is the number of electrons involved in the redox process, F is Faraday's constant and $\rho$ is the density of the NP material.

The comparison of the histogram obtained by nano-impact analysis against that gained from SEM images can be seen in Figure 4. The SEM image used for sizing is that shown in Figure 1. Strong agreement between the two particle distributions can be seen, with the mean spherical radius of the particles being (48 \pm 1$) \mathrm{nm}$ and $(46 \pm 1) \mathrm{nm}$ and standard deviations of $14 \mathrm{~nm}$ and $22 \mathrm{~nm}$ respectively. The close agreement between electrochemical and SEM sizing of the AgBr NPs strongly indicates that the NPs formed are pure AgBr. This is because any Ag already present in the NP would reduce the amount of $\mathrm{AgBr}$ available for electrochemical reduction and 
would lead to an underestimate of the NP size. This is further confirmed by independent UV-Vis characterisation of the NPs which can be seen in Figure 5. Ag NPs are well known to exhibit a characteristic peak at $\sim 400 \mathrm{~nm}$ [22]. Such a peak is clearly absent from the observed UV-Vis spectrum obtained from the AgBr NPs showing the NPs formed are not Ag.

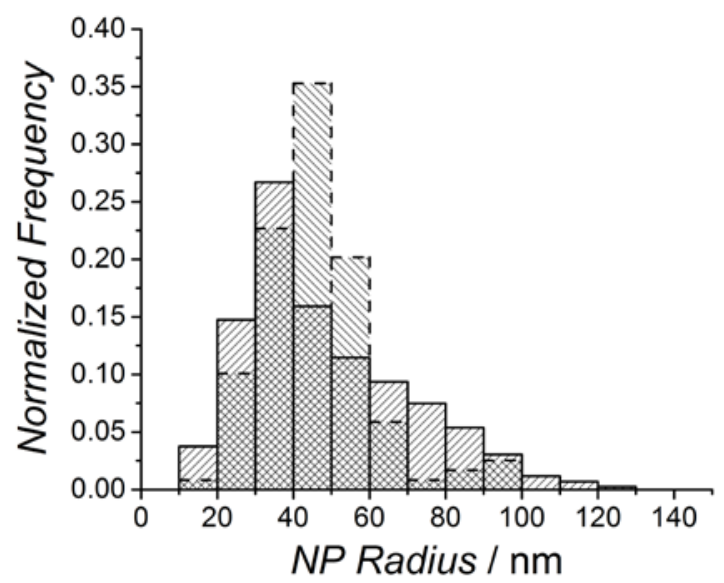

Figure 4. Nanoparticle size distribution comparison between SEM (dashed outline) and nanoparticle impacts (solid outline).

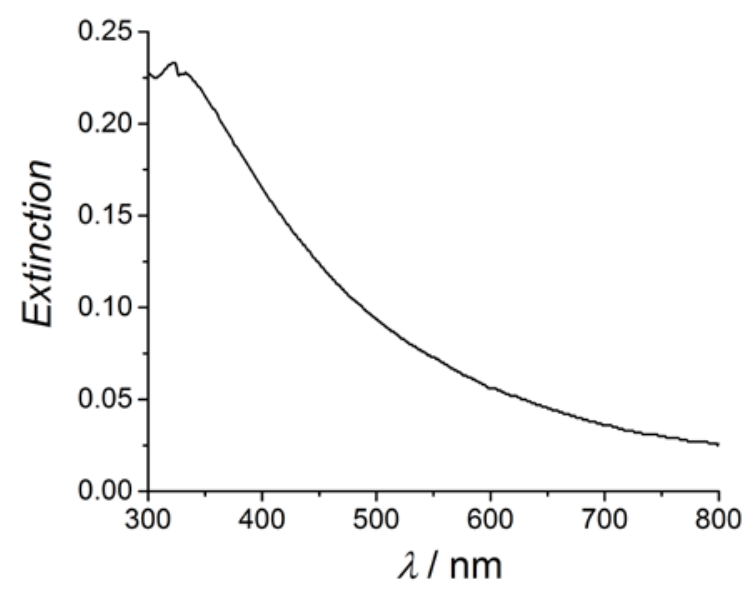

Figure 5. UV-Vis characterisation of AgBr NPs. 
To establish the timescale of conversion of the NPs with ascorbic acid, the reaction was first investigated by UV-Vis spectrometry. A $30 \mathrm{~mL}$ solution of $0.3 \mathrm{mM}$ ascorbic acid in $0.10 \mathrm{M} \mathrm{pH}$ 10 buffer was prepared in glass cell with quartz window. To this, $6 \mathrm{uL}$ of the NP stock suspension was added and the solution bubbled with nitrogen to prevent oxidation of the ascorbic acid. The cell solution was maintained under constant illumination and samples removed every 10 mins for UV-Vis analysis. Figure 6 shows the UV-Vis spectrum of the NPs over a 60 min period.

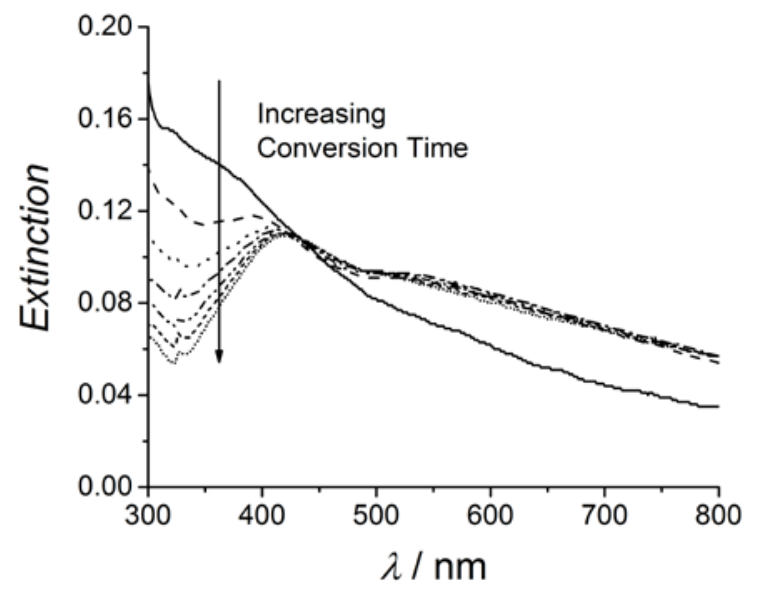

Figure 6. UV-Vis tracking of AgBr NP conversion over 60 minutes. Measurements taken at 10 minute intervals from 0 to 60 minutes.

A clear trend can be seen with the emergence of a broad peak at $422 \mathrm{~nm}$, stabilising after 50 mins. The peak formed is indicative of the formation of large Ag NPs with a wide size distribution [22], matching that of the originating AgBr nanoparticles.

Next, the photochemical conversion mechanism was probed voltammetrically by nano-impact experiments, studying both the rate of formation of $\mathrm{Ag}$ and the loss of AgBr. Current-time transients of $50 \mathrm{sec}$ were recorded after full conversion by ascorbic acid (60 mins) at oxidative overpotentials of $+0.40 \mathrm{~V}$ and $+0.60 \mathrm{~V}$ vs MSE to try to detect the formed Ag NPs. No faradaic 
spikes were detected after full conversion despite the confirmation of suspended Ag NPs through UV-Vis spectrometry. This was attributed to reduction in size of the formed Ag NPs due to the respective density differences of $\mathrm{AgBr}$ and Ag. The decrease in size of the particle could occur without change to the gelatine layer thickness, and would result in an increased distance between the particle and electrode on impact when compared to that of the original $\mathrm{AgBr} \mathrm{NP}$. Additionally, hypobromite which can be formed during the synthesis process in alkaline conditions has been shown to cause gelatine cross-linking [23], which may be an additional barrier for Ag NP oxidation. The absence of impacts at overpotential of $+520 \mathrm{mV}$ represents a significant kinetic barrier to Ag NP oxidation under these experimental conditions. Complementary stripping voltammetry conducted on AgBr NPs converted by ascorbic acid to detect the presence of Ag NPs resulted in interference by ascorbic acid oxidation. This was found to mask the Ag oxidation signal and meant no analysis of the change in Ag oxidation peak could be observed.

To study the loss of $\mathrm{AgBr}$ in the NPs during photochemical conversion, current-time transients with $50 \mathrm{sec}$ duration were conducted at $10 \mathrm{~min}$ time intervals with the potential held at $-1.00 \mathrm{~V}$ vs MSE. Each timed measurement was carried out individually such that illumination was constant until measurements were recorded. This was necessary as the light source contributed noise to the system and was switched off during the measurement. The results are summarised in Figure 7, which shows example current-time transients across the conversion time period. A trend can be seen with a reduction in spike size over the conversion timescale, with no spikes observed at 60 mins. Decreasing spike charge over time is consistent with conversion of the particles from $\mathrm{AgBr}$ to $\mathrm{Ag}$ and the loss of $\mathrm{AgBr}$ available for electrochemical reduction in converting particles. 


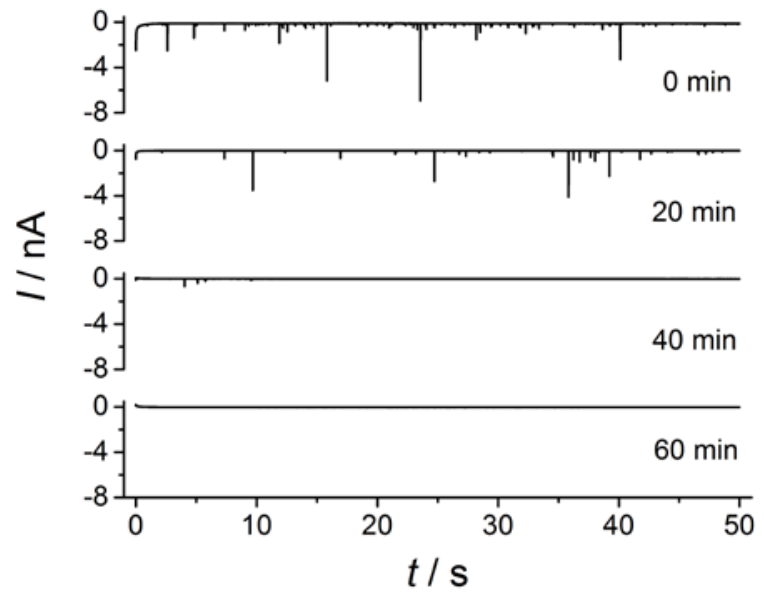

Figure 7. Current-time transients showing the change in AgBr reductive impacts over the time scale of conversion. This was conducted in $0.1 \mathrm{mM}$ AgBr NP and $0.3 \mathrm{mM}$ ascorbic acid in 0.10 $\mathrm{M}$ pH 10 buffer at $-1.00 \mathrm{~V}$ vs MSE.

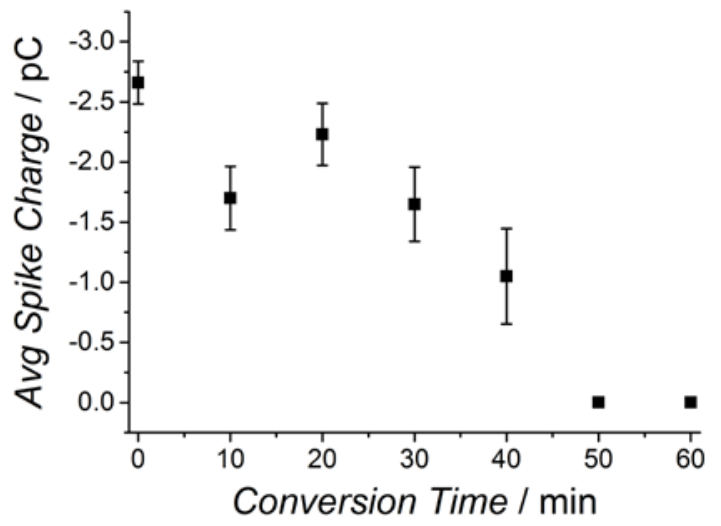

Figure 8. Average reductive spike charge as a function of conversion time.

The spike charges measured over the duration of conversion were analysed and plotted against conversion time to give Figure 8. A general trend can be clearly seen, with a decreasing value for the average spike charge over the course of conversion. This decreased until 50 mins, where no spikes are recorded. This decrease in spike charge is associated with the loss of $\mathrm{AgBr}$ as the conversion progresses and matches the timescale for conversion as obtained by UV-Vis 
spectrometry. This shows that by analysing the average spike charge, it is possible to track the conversion of NPs to its completion.

If a shell of Ag were to have formed the release of halide would have been halted due to a compact outer layer. Without a means of expelling the halide, a build-up of $\mathrm{Br}^{-}$in the centre of the NP would promote recombination of the photochemically generated $\mathrm{Ag}^{+}$ions with adjacent $\mathrm{Br}^{-}$ions to form $\mathrm{AgBr}^{[14]}$. This would result in an abrupt fall in reductive spike charge as soon as this shell developed. After 40 mins, a $60.5 \%$ loss of NP volume can be measured which can be expressed as the conversion of more than $10 \mathrm{~nm}$ of the outer layer of the NP. A layer of this thickness would provide a significant barrier for $\mathrm{Br}^{-}$release from the core. Instead, a gradual reduction in spike charge occurs, indicating that $\mathrm{AgBr}$ is electrochemically accessible throughout the conversion process. This allows for expulsion of the halide and continued conversion of the NP. Therefore, this suggests that the photochemical synthesis of AgBr occurs through an irregular mechanism extending to the core of the particle before the entirety of the surface is converted.

\section{CONCLUSIONS}

The nano-impact technique has been shown as an informative tool for probing the synthesis mechanism of $\mathrm{AgBr}$ NPs to Ag through the photochemical reduction by ascorbic acid. By measuring the change in the amount of $\mathrm{AgBr}$ electrochemically accessible throughout the conversion timescale the reaction mechanism was found to be of an irregular nature, propagating over the NP structure. This work has expanded the use of the nano-impact technique and clearly demonstrates its use in the field of NP synthesis for both mechanistic investigation and tracking of synthesis. The use of this technique would be especially applicable for the tracking of NP 
conversion where identifiable UV-Vis peaks are not available, or not measurable as in opaque media [24].

\section{AUTHOR INFORMATION}

\section{Corresponding Author}

* Prof. Richard G. Compton, Department of Chemistry, Physical and Theoretical Chemistry Laboratory, Oxford University, South Parks Road, Oxford, OX1 3QZ, United Kingdom, Richard.Compton@chem.ox.ac.uk

\section{ACKNOWLEDGMENT}

We acknowledge funding from the European Research Council (ERC) under the European Union’s Seventh Frame-work Program (FP/2007-2013)/ERC Grant Agreement no. [320403].

\section{REFERENCES}

[1] M. Murphy, K. Ting, X. Zhang, C. Soo, Z. Zheng, Am. J. Nanomater. 2015, vol. 2015, p. 1-12.

[2] F. W. Campbell, R. G. Compton, Anal. Bioanal. Chem. 2009, vol. 396, p. 241-259.

[3] N. L. Pacioni, C. D. Borsarelli, V. Rey, A. V. Veglia, Synthetic Routes for the Preparation of Silver Nanoparticles, Switzerland: Springer International Publishing, 2015, p. 13-46.

[4] C. B. Murray, C. R. Kagan, M. G. Bawendi, Annu. Rev. Mater. Sci. 2000, vol. 30, p. 545610. 
[5] H. Hiramatsu, F. E. Osterloh, Chem. Mater. 2004, vol. 16, p. 2509-2511.

[6] J. Eastoe, M. J. Hollamby, L. Hudson, Adv. Colloid Interface Sci. 2006, vol. 128-130, p. $5-15$.

[7] A. A. Isse, S. Gottardello, C. Maccato, A. Gennaro, Electrochem. Commun. 2006, vol. 8, p. $1707-1712$.

[8] V. K. LaMer, R. H. Dinegar, J. Am. Chem. Soc. 1950, vol. 72, p. 4847-4854.

[9] Y. De Smet, L. Deriemaeker, R. Finsy, Langmuir 1997, vol. 13, p. 6884-6888.

[10] H. Y. Zhu, Y. Lan, X. P. Gao, S. P. Ringer, Z. F. Zheng, D. Y. Song, J. C. Zhao, J. Am. Chem. Soc. 2005, vol. 127, p. 6730-6736.

[11] Z. Tang, Y. Wang, K. Sun, N. A. Kotov, Adv. Mater. 2005, vol. 17, p. 358-363.

[12] A. Król-Gracz, E. Michalak, P. M. Nowak, A. Dyonizy, Cent. Eur. J. Chem. 2011, vol. 9, p. 982-989.

[13] A. Król-Gracz, P. Nowak, E. Michalak, A. Dyonizy, Acta Phys. Pol., A. 2012, vol. 121, p. 196.

[14] T. B. Brill, Light, its interaction with art and antiquities, New York: Plenum Press, 1980, p. 258.

[15] Y. G. Zhou, N. V. Rees, R. G. Compton, Angew. Chem., Int. Ed. Engl. 2011, vol. 50, p. 4219-4221.

[16] X. Xiao, A. J. Bard, J. Am. Chem. Soc. 2007, vol. 129, p. 9610-9612.

[17] A. Fernando, S. Parajuli, M. A. Alpuche-Aviles, J. Am. Chem. Soc. 2013, vol. 135, p. 10894-10897.

[18] H. S. Ahn, A. J. Bard, Angew. Chem., Int. Ed. Engl. 2015, vol. 127, p. 13957 
[19] C. Batchelor-McAuley, J. Ellison, K. Tschulik, P. L. Hurst, R. Boldt, R. G. Compton, Analyst. 2015, vol. 140, p. 5048-5054.

[20] T. R. Bartlett, S. V. Sokolov, R. G. Compton, ChemistryOpen. 2015, vol. 4, p. 600-605.

[21] W. Cheng, X. F. Zhou, R. G. Compton, Angew. Chem., Int. Ed. Engl. 2013, vol. 52, p. 12980-12982.

[22] D. Paramelle, A. Sadovoy, S. Gorelik, P. Free, J. Hobley, D. G. Fernig, Analyst. 2014, vol. 139, p. 4855.

[23] A. W. Kenchington, Biochem. J. 1957, vol. 68, p. 458-468.

[24] H. S. Toh, R. G. Compton, ChemistryOpen. 2015, vol. 4, p. 261-263. 